\title{
Refuting a Conjecture of Goemans on Bounded Degree Spanning Trees
}

\author{
S. Chestnut ${ }^{\mathrm{a}}$, M. Nägele ${ }^{\mathrm{a}, *}$, R. Zenklusen ${ }^{\mathrm{a}}$
}

${ }^{a}$ Department of Mathematics, ETH Zurich, Rämistrasse 101, 8092 Zurich, Switzerland

\begin{abstract}
In 2006, Goemans presented an approximation algorithm for the minimum bounded degree spanning tree problem that constructs a tree with cost at most the optimal value of an LP relaxation but degree bound violations of up to two units per vertex. He conjectured that violations of at most one per vertex are attainable, providing a second conjecture that would make his approach achieve this guarantee. While the first conjecture was answered positively by Singh and Lau, we refute the second.
\end{abstract}

Keywords: bounded degree spanning tree, approximation algorithm, matroid

\section{Introduction}

The minimum bounded degree spanning tree (MBDST) problem is the following: Given a graph $G=(V, E)$, costs $c: E \rightarrow \mathbb{R}$ and degree bounds $d: V \rightarrow \mathbb{Z}_{>0}$, find a minimum cost spanning tree $T$ of $G$ such that for all $v \in V$, the degree of $v$ in $T$ is at most $d(v)$.

It is well known that for any $D \geq 2$, MBDST is NP-hard even if all vertices have the same degree bound $d(v)=D$. In particular, for $D=2$, the problem asks to find a Hamiltonian path. Thus interest arose in finding approximation algorithms with various trade-offs between cost of the spanning tree and violation of the degree bounds. While for the unweighted problem, the best possible approximation with degree bound violations of only at most one unit was found by Fürer and Raghavachari [1] in 1994, the analogous result for the weighted case remained open until 2007.

After a series of papers [2, 3, 4, 5, 6, 7] made progress on the approximation guarantee of the weighted problem, Goemans [8] presented the first algorithm that returned a spanning tree violating the degree constraints by at most an additive constant, namely +2 , and of cost no more than the optimal spanning tree that does not violate any degree constraint. The gap to degree bound violations of at most one unit per vertex for the weighted case was closed by Singh and Lau using an iterative relaxation technique [9]. There are many constrained spanning tree problems that are closely related to MBDST, and also generalize it in several ways (see [10, 11, 12, 13, 14, 15] and references therein).

In this work, we refute a conjecture of Goemans that would allow for improving his approach in [8] to degree bound violations of at most one unit per vertex. Even though Singh and Lau [9] already obtained an optimal approximation algorithm through different techniques, such an extension of Goemans' algorithm would have been interesting. In particular, Goemans'

\footnotetext{
${ }^{*}$ Corresponding author.

Email addresses: stephenc@ethz.ch (S. Chestnut), martinn@ethz.ch (M. Nägele), ricoz@math.ethz.ch (R. Zenklusen)
}

approach shows that any solution to the natural LP relaxation of MBDST is contained in a matroid intersection polytope, all of whose vertices correspond to spanning trees violating each degree constraint by at most +2 . This, for example, provides an easy way to decompose any LP solution as a convex combination of spanning trees with violation at most +2 . Goemans' conjecture being true would have automatically extended these structural results to constraint violation of at most +1 , which is best possible. We start by a brief description of Goemans' approach before stating and refuting his conjecture.

Goemans' approach considers the natural LP relaxation of MBDST, given by

$$
\begin{array}{cl}
\min \quad c^{\top} x & \\
\text { s.t. } x(E[S]) & \leqslant|S|-1 \quad \forall S \subset V, S \neq \emptyset, \\
x(E) & =|V|-1, \\
x(\delta(v)) & \leqslant d(v) \quad \forall v \in V, \\
x & \in \mathbb{R}_{\geqslant 0}^{E} .
\end{array}
$$

$\left(\mathrm{LP}_{\mathrm{MBDST}}\right)$

Here, $E[S]$ denotes the set of edges inside $S, \delta(v)$ denotes the set of edges incident to vertex $v$ and $x(U)=\sum_{e \in U} x(e)$ for $U \subseteq E$. Based on this, Goemans' algorithm proceeds as follows:

1. Obtain an optimal vertex solution $x^{*}$ to (LPMBDST), and let $E^{*}=\operatorname{supp}\left(x^{*}\right)=\{e \in E \mid x(e)>0\}$.

2. Orient the edges of the graph $\left(V, E^{*}\right)$ to obtain a directed graph $\left(V, A^{*}\right)$ such that all indegrees are at most two.

3. Using matroid intersection, compute a minimum spanning tree within $E^{*}$, i. e., a basis of the graphic matroid $M_{1}$ over $E^{*}$, that is simultaneously independent in the partition matroid $M_{2}$ whose independent sets are given by $\mathcal{I}=\left\{F \subseteq E^{*}|| F \cap \delta_{A^{*}}^{+}(v) \mid \leqslant d(v) \quad \forall v \in V\right\}$.

Here, $\delta_{A^{*}}^{+}(v)$ denotes the set of edges going out of $v$ in the oriented graph $\left(V, A^{*}\right)$. The key result that Goemans proves for obtaining the orientation is that $\left(V, E^{*}\right)$ is sparse in the sense that for all non-empty $U \subseteq V$, we have $\left|E^{*}[U]\right| \leqslant 2|U|-3$. 
This property implies that an orientation as described in step 2 exists. Moreover, such an orientation can be found efficiently. The orientation guarantees that the tree returned by the matroid intersection violates the degree bounds by at most two units: At every vertex $v \in V$, at most $d(v)$ of the outgoing edges appear in $T$, and there are at most two additional incoming edges, which leads to a total of at most $d(v)+2$ edges in $T$ that may be incident with $v$.

Therefore, the crucial step in Goemans' approach is to get a good orientation of the graph $\left(V, E^{*}\right)$ that allows for a relatively accurate description of the degree constraints by a matroid. This idea leads to the following conjecture on the existence of an even better orientation.

Conjecture 1 (Goemans, [8]). Let $x^{*}$ be a vertex solution of $\left(\mathrm{LP}_{\mathrm{MBDST}}\right)$, and let $E^{*}=\operatorname{supp}\left(x^{*}\right)$. Then, there exists an orientation $A^{*}$ of $E^{*}$ such that for all $v \in V$, we have

$$
\sum_{e \in \delta_{A^{*}}^{-}(v)}\left(1-x^{*}(e)\right) \leqslant 1 .
$$

As Goemans [8] showed, if this conjecture was true, the matroid $M_{2}$ could be replaced by a different partition matroid with independent sets $\mathcal{I}=\left\{F \subseteq E^{*}|| F \cap \delta_{A^{*}}^{+}(v) \mid \leqslant\left\lceil x^{*}\left(\delta_{A^{*}}^{*}(v)\right)\right\rceil \forall v \in\right.$ $V$, leading to a spanning tree that violates the degree bounds by at most one unit.

We are able to refute an even weaker version of Conjecture 1 . Let $z^{*}:=1-x^{*}$ denote the spare vector, then the conjecture states that for every edge $e$, the spare $z^{*}(e)$ can be assigned to one of the incident vertices such that in total, each vertex gets at most one unit of spare assigned to it. We show that even if we allow splitting the spare of every edge among the incident vertices, this is impossible. To do so, we construct an instance containing what we call an obstruction: A set $U \subseteq V$ such that $z^{*}(E[U])>|U|$, i. e., there is a subgraph on $|U|$ vertices with more than $|U|$ units of spare on the induced edges. This clearly contradicts even the weakening of Conjecture 1.

The paper is organised as follows. In Section 2, we generalise the problem to rational degree bounds and construct a family of instances giving counterexamples of the prescribed type. Section 3 then explains how fractional degree bounds can be modelled in larger instances by attaching certain auxiliary graphs to vertices with fractional degree bounds.

For notational convenience, we define two polytopes associated to a graph $G=(V, E)$ and degree bounds $d: V \rightarrow \mathbb{Q}$, namely the spanning tree polytope $P_{S T}(G)$ and the bounded degree spanning tree polytope $P_{B D S T}(G, d)$, given by

$$
\begin{gathered}
P_{S T}(G):=\left\{x \in \mathbb{R}_{\geqslant 0}^{E} \mid \begin{array}{c}
x(E[S]) \leqslant|S|-1 \forall S \subset V, S \neq \emptyset, \\
x(E)=|V|-1
\end{array}\right\}, \\
P_{B D S T}(G, d):=\left\{x \in P_{S T}(G) \mid x(\delta(v)) \leqslant d(v) \forall v \in V\right\} .
\end{gathered}
$$

Note that while $P_{S T}$ is integral for all $G$ (see [16]), $P_{B D S T}$ is not, in general. Constraints of the form $x(E[S]) \leqslant|S|-1$ and $x(\delta(v)) \leqslant d(v)$ are referred to as spanning tree constraints and degree constraints, respectively.

\section{Counterexamples with Fractional Degree Bounds}

For $k \in \mathbb{Z}_{>0}$ and $\varepsilon \in(0,1 / 2) \cap \mathbb{Q}$, let the graph $G=(V, E)$ on the vertex set $V:=\left\{u_{1}, \ldots, u_{k-1}\right\} \cup\left\{v_{1}, \ldots, v_{k}\right\}$ be as in Figure 1, where we also indicate a point $x^{*} \in \mathbb{R}_{\geqslant 0}^{E}$ defined by

$$
x^{*}(e):= \begin{cases}\varepsilon & \text { if } \exists i, j \in[k]: e=v_{i} v_{j}, \\ 1-\varepsilon & \text { if } e=u_{1} v_{2}, \\ 1-2 \varepsilon & \text { if } \exists i \in\{2, \ldots, k-1\}: e=u_{i} v_{i+1}, \\ 1 & \text { if } e=v_{1} u_{1} \text { or } \exists i \in[k-2]: e=u_{i} u_{i+1} .\end{cases}
$$

Moreover, we define $d(v):=x^{*}(\delta(v))$ for all $v \in V$.

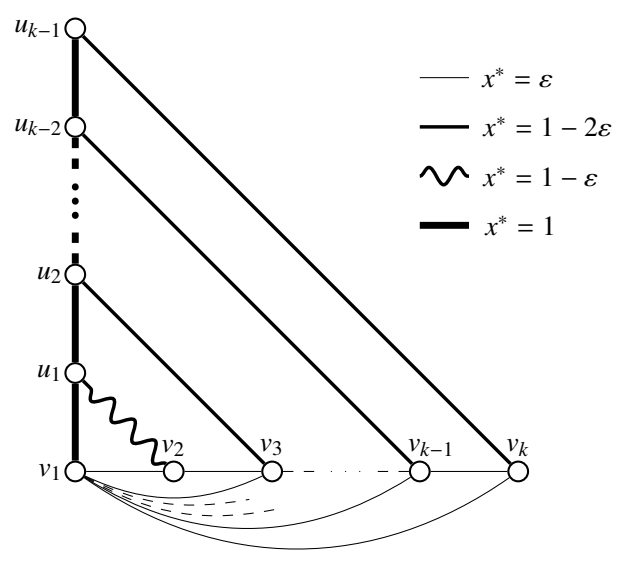

Figure 1: The graph $G$ and the point $x^{*} \in \mathbb{R}^{E}$.

We now show that $x^{*}$ is an extreme point of $P_{B D S T}(G, d)$.

Lemma 2. For $G=(V, E), d: V \rightarrow \mathbb{Q}$ and $x^{*} \in \mathbb{R}_{\geqslant 0}^{E}$ as defined above, $x^{*}$ is an extreme point of $P_{B D S T}(G, d)$.

Before proving the lemma, we observe that for any $k \geqslant 4$ and $\varepsilon<\frac{k-3}{2 k-3}$, the extreme point $x^{*}$ contains an obstruction, namely the set $U=\left\{v_{1}, \ldots, v_{k}\right\}$. Indeed, using the assumptions on $k$ and $\varepsilon$, we see that the spare $z^{*}$ satisfies

$$
z^{*}(E[U])=(2 k-3)(1-\varepsilon)>k=|U| .
$$

Hence, distributing spares in accordance with Conjecture 1 is impossible. To refute Goemans' conjecture, it suffices to show that we can "simulate" the fractional degree bounds $d$ used to obtain the extreme point $x^{*}$ with integral degree bounds (as a subsystem of a larger instance). This will be done in section 3 .

Proof of Lemma 2. We first show that $x^{*} \in P_{B D S T}(G, d)$. The degree constraints $x(\delta(v)) \leqslant d(v)$ are satisfied at $x=x^{*}$ by definition of $d$ for all $v \in V$, so it suffices to see $x^{*} \in P_{S T}(G)$. Equivalently, it suffices to prove that $x^{*}$ can be written as a convex combination of indicator vectors of spanning trees of $G$. This can be achieved using the trees in Figure 2 with the indicated coefficients.

Knowing $x^{*} \in P_{B D S T}(G, d)$, it is enough to reveal a fullrank system of constraints that are tight at $x^{*}$. By definition of $d$, all degree constraints $x(\delta(v)) \leqslant d(v)$ are tight at $x^{*}$. However, we only use those for $v \in V \backslash\left\{v_{1}\right\}$. Among the spanning tree 

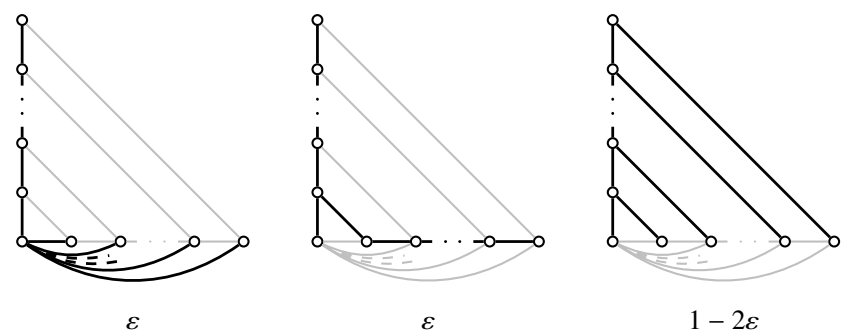

Figure 2: Decomposition of $x^{*}$ as convex combination of spanning trees.

constraints $x(E[S]) \leqslant|S|-1$, we see that choosing for $S$ the endpoints of one of the edges $v_{1} u_{1}, u_{1} u_{2}, \ldots, u_{k-2} u_{k-1}$, or a set of the form $U_{i}:=\left\{v_{1}, \ldots, v_{i+1}, u_{1}, \ldots, u_{i}\right\}$, gives a constraint that is tight at $x^{*}$. These tight constraints are illustrated in Figure 3.

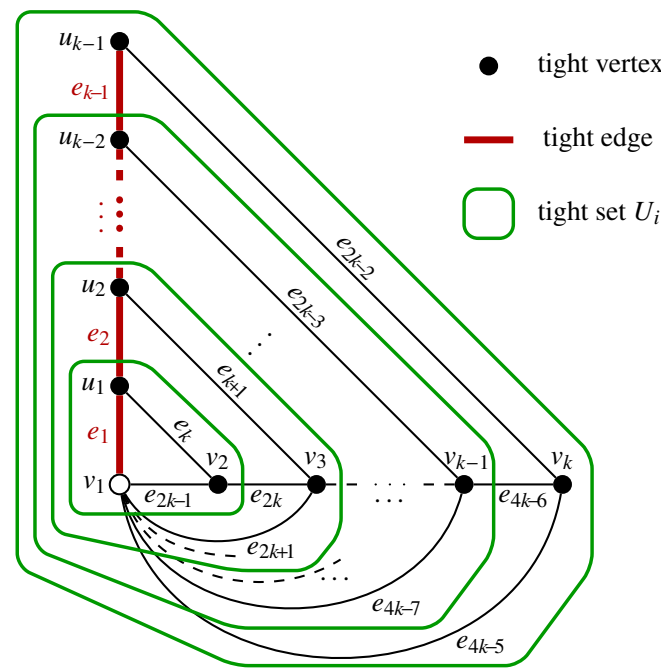

Figure 3: A selection of tight constraints at $x^{*}$ and a numbering of the edges.

We claim that if we number the edges as indicated in Figure 3, we can find an order of the listed tight constraints such that the matrix with indicator vectors of the constraints as rows is a lower triangular square matrix with ones on the diagonal, proving that the chosen subsystem is of full rank. This can be achieved by numbering the constraints as follows:

Constraint 1: Tight edge $v_{1} u_{1}=e_{1}$.

Constraint i for $i \in\{2, \ldots, k-1\}$ : Tight edge $u_{i-1} u_{i}=e_{i}$.

Constraint $k+i-1$ for $i \in[k-1]$ : Degree constraint at $u_{i}$, containing the edges $e_{i+1}, e_{i}, e_{k+i-1}$ (only the last two at $\left.u_{k-1}\right)$.

Constraint $2(k+i)-3$ for $i \in[k-1]$ : Tight set $U_{i}$, containing the edges $e_{1}, \ldots, e_{i}, e_{k}, \ldots, e_{k+i-1}$ and $e_{2 k-1}, \ldots, e_{2(k+i)-3}$.

Constraint $2(k+i)-2$ for $i \in[k-2]$ : Degree constraint at $v_{i+1}$, containing the edges $e_{2(k+i)-4}, e_{k+i-1}, e_{2(k+i)-3}, e_{2(k+i)-2}$ (only the last three at $v_{2}$ ).

Note that the constraints corresponding to tight sets $U_{i}$ and the degree constraints at vertices $v_{i}$ appear alternatingly in this order. The different types of constraints and the edges they in- clude are shown again in Figure 4 (except for the obvious case of tight edges).

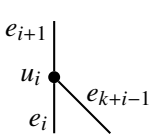

$\delta\left(u_{i}\right)$

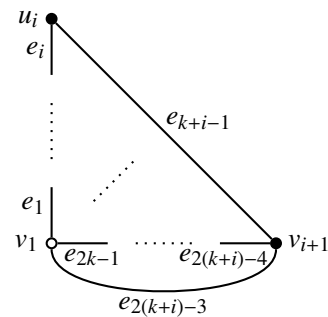

$E\left[U_{i}\right]$

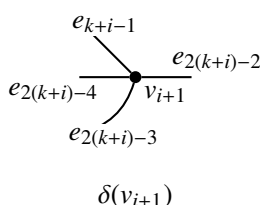

$\delta\left(v_{i+1}\right)$
Figure 4: Constraints and the underlying sets of edges.

From the above list, it is clear that the desired property holds. This proves Lemma 2.

\section{Modelling Fractional Degree Bounds}

\subsection{Attaching Auxiliary Graphs}

The goal of this section is to show how an extreme point of a bounded degree spanning tree polytope $P_{B D S T}(G, d)$ with non-integral degree bounds $d$ can be obtained as a restriction of a vertex to the relaxation of a larger instance with integral degree bounds. We say that a non-negative rational number $r$ is an extremal degree slack if there exists a graph $G$, integral degree bounds $d$ and an extreme point $x \in P_{B D S T}(G, d)$ such that for some $v \in V$, we have $r=d(v)-x(\delta(v))$, i. e., $r$ is the slack of the degree constraint at $v$. In this case, we call the instance $(G, d, x, v)$ a certifying instance for $r$. We let $\mathcal{D} \subseteq \mathbb{Q} \geqslant 0$ denote the set of all extremal degree slacks. The next lemma shows how certifying instances can be used to model fractional degree bounds by integral ones. For a real number $x$, we denote by $\operatorname{frac}(x):=x-\lfloor x\rfloor$ the fractional part of $x$.

Lemma 3. Let $G_{1}=\left(V_{1}, E_{1}\right)$ be a graph, $d_{1}: V_{1} \rightarrow \mathbb{Q}$, and $x_{1}$ an extreme point of $P_{B D S T}\left(G_{1}, d_{1}\right)$. If for all $v \in V_{1}$, we have $\operatorname{frac}\left(d_{1}(v)\right) \in \mathcal{D}$, then we can construct a graph $G=(V, E)$ that contains $G_{1}$ as an induced subgraph and $d: V \rightarrow \mathbb{Z}$ such that there exists an extreme point $x \in P_{B D S T}(G, d)$ with $\left.x\right|_{E_{1}}=x_{1}$.

Proof. Let $v_{1} \in V_{1}$ be such that $d_{1}\left(v_{1}\right) \notin \mathbb{Z}$. We show how to model this single fractional degree bound by an integral one. If the degree constraint at $v_{1}$ is not tight with respect to $x_{1}$, we can simply round $d_{1}\left(v_{1}\right)$ up without changing the property that $x_{1}$ is an extreme point of $P_{B D S T}\left(G_{1}, d_{1}\right)$. Thus, we assume tightness of this degree constraint, i. e., $x\left(\delta_{G_{1}}\left(v_{1}\right)\right)=d_{1}\left(v_{1}\right)$.

By assumption, $\operatorname{frac}\left(d_{1}(v)\right) \in \mathcal{D}$, so there exists a certifying instance $\left(G_{2}=\left(V_{2}, E_{2}\right), d_{2}, x_{2}, v_{2}\right)$ for frac $\left(d_{1}(v)\right)$. Define a new graph $G:=G_{1} \cup \dot{\cup} G_{2} / v_{1} \sim v_{2}$ obtained by taking the union of $G_{1}$ and $G_{2}$ and identifying the vertices $v_{1}$ and $v_{2}$ in a new vertex $v$, and let $x \in \mathbb{R}^{E[G]}$ and $d: V[G] \rightarrow \mathbb{Q}$ be defined by

$$
\begin{aligned}
& \left.x\right|_{E_{1}}:=x_{1}, \\
& \left.x\right|_{E_{2}}:=x_{2} \text {, } \\
& \left.d\right|_{V_{1} \backslash\left\{v_{1}\right\}}:=d_{1}, \\
& \text { and } \\
& \left.d\right|_{V_{2} \backslash\left\{v_{2}\right\}}:=d_{2}, \\
& d(v):=\left\lfloor d_{1}\left(v_{1}\right)\right\rfloor+d_{2}\left(v_{2}\right) .
\end{aligned}
$$


With these definitions, $\left(G_{1}, d_{1}, x_{1}\right)$ and $\left(G_{2}, d_{2}, x_{2}\right)$ complement each other with the only modification being the modified degree bound at the new vertex $v=v_{1}=v_{2}$, see Figure 5. Moreover, $G$ contains $G_{1}$ as an induced subgraph.

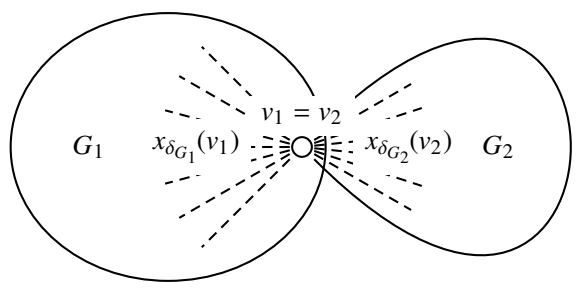

Figure 5: Modelling one fractional degree bound.

We claim that $x$ is an extreme point of $P_{B D S T}(G, d)$. As before, we first check feasibility of $x$ for $P_{B D S T}(G, d)$. The degree bounds at vertices different from $v$ are directly implied by the corresponding constraints in $P_{B D S T}\left(G_{1}, d_{1}\right)$ and $P_{B D S T}\left(G_{2}, d_{2}\right)$, and at vertex $v$, we have

$$
\begin{aligned}
x(\delta(v)) & =x\left(\delta(v) \cap E_{1}\right)+x\left(\delta(v) \cap E_{2}\right)=x_{1}\left(\delta\left(v_{1}\right)\right)+x_{2}\left(\delta\left(v_{2}\right)\right) \\
& =d_{1}\left(v_{1}\right)+d_{2}\left(v_{2}\right)-\operatorname{frac}\left(d_{1}\left(v_{1}\right)\right)=\left\lfloor d_{1}\left(v_{1}\right)\right\rfloor+d_{2}\left(v_{2}\right) \\
& =d(v),
\end{aligned}
$$

using $E=E_{1} \dot{\cup} E_{2}$ and the assumption that the degree bound at $v_{2}$ has slack frac $\left(d_{1}\left(v_{1}\right)\right)$. Moreover, we also have $x \in P_{S T}(G)$. This follows from the fact that, by construction, spanning trees in $G$ are disjoint unions of a spanning tree in $G_{1}$ and a spanning tree in $G_{2}$, which implies that $P_{S T}(G)$ is the direct sum of $P_{S T}\left(G_{1}\right)$ and $P_{S T}\left(G_{2}\right)$. Now $x \in P_{S T}(G)$ follows since $x$ is the direct sum of $x_{1} \in P_{S T}\left(G_{1}\right)$ and $x_{2} \in P_{S T}\left(G_{2}\right)$.

To see that $x$ is an extreme point of $P_{B D S T}(G, d)$, we need to reveal a system of constraints which, if set to equality, has $x$ as a unique solution. For both $i \in\{1,2\}$ and suitable $k_{i} \in \mathbb{Z}$, let $A_{i} \in \mathbb{Z}^{k_{i} \times\left|E_{i}\right|}$ and $b_{i} \in \mathbb{Z}^{k_{i}}$ be such that the systems

$$
\left\{\begin{array} { r l } 
{ A _ { 1 } y _ { 1 } } & { = b _ { 1 } , } \\
{ y _ { 1 } ( \delta _ { G _ { 1 } } ( v _ { 1 } ) ) } & { = d _ { 1 } ( v _ { 1 } ) , } \\
{ y _ { 1 } } & { \in \mathbb { R } ^ { E _ { 1 } } }
\end{array} \quad \text { and } \quad \left\{\begin{array}{rl}
A_{2} y_{2} & =b_{2}, \\
y_{2} & \in \mathbb{R}^{E_{2}}
\end{array}\right.\right.
$$

are the systems of all tight constraints for $x_{i}$ in $P_{B D S T}\left(G_{i}, d_{i}\right)$, respectively. Note that we know by assumption that the explicitly indicated degree constraint at $v_{1}$ is tight at $x_{1}$. Moreover, as the $x_{i}$ are extreme points, we also know that both systems are of full column rank. We claim that the system

$$
\left(\begin{array}{cc}
A_{1} & 0 \\
\chi_{G_{1}}\left(v_{1}\right) & \chi^{\delta_{G_{2}}\left(v_{2}\right)} \\
0 & A_{2}
\end{array}\right) y=\left(\begin{array}{c}
b_{1} \\
d(v) \\
b_{2}
\end{array}\right)
$$

for $y \in \mathbb{R}^{E}$ is a system of constraints from $P_{B D S T}(G, d)$ set to equality, and it has $x$ as a unique solution. To see this, let $A$ be the matrix in (3). Note that by construction of $G$ from $G_{1}$ and $G_{2}$, every row of $A$ is of the form $y(E[S])=|S|-1$ for some $S \subseteq V$, or $y\left(\delta_{G}(u)\right)=d(u)$ for some $u \in V$. Moreover, (1) and (2) imply that $x$ satisfies the system (3). The fact that the systems in (2) have full column rank directly implies that $A$ has full column rank, too, so $x$ is indeed the unique solution of (3). This proves that $x$ is an extreme point of $P_{B D S T}(G, d)$.

Note that the number of fractional components of $d$ is precisely one less than the number of fractional components in $d_{1}$, so by iterating the construction, we can remove one fractional degree bound after another, always maintaining the requirements that $\left.x\right|_{E_{1}}=x_{1}$ and that $G_{1}$ appears as an induced subgraph of the resulting graph.

\subsection{Constructing Extremal Degree Slacks}

By Lemma 3, we only need to prove that the set $\mathcal{D}$ of extremal degree slacks is sufficiently large for our construction to work. In fact, we prove the following theorem.

Theorem 4. Any non-negative rational number is an extremal degree slack, i. e., $\mathcal{D}=\mathbb{Q} \geqslant 0$.

It is easy to see that $\mathbb{Z}_{\geqslant 0} \subseteq \mathcal{D}$, for example by taking a 2vertex graph with large enough integral degree bounds. Moreover, $r \in \mathcal{D}$ if and only if $\operatorname{frac}(r) \in \mathcal{D}$ : In a certifying instance for $r$, simply reduce the degree bound with slack $r$ by $\lfloor r\rfloor$ and note that this works in the other direction as well. Using this observation, the following two lemmas suffice for constructing certifying instances for any non-negative rational extremal degree slack.

Lemma 5. For every $p \in \mathbb{Z}_{\geqslant 2}$, we have $\frac{p-1}{p} \in \mathcal{D}$.

Proof. Fix an integer $p \in \mathbb{Z}_{\geqslant 2}$. We construct a certifying instance for the degree bound slack $\frac{p-1}{p}$. We consider the graph $G(V, E)$ as illustrated in Figure 6, with vertex set $V=\{s\} \cup$ $\left\{u_{1}, \ldots, u_{p-1}\right\} \cup\left\{v_{1}, \ldots, v_{p}\right\} \cup\left\{w_{1}, \ldots, w_{p-1}\right\}$, and, for $\varepsilon \in \mathbb{R}$, the family of vectors $x(\varepsilon) \in \mathbb{R}^{E}$ as shown in the same figure. Let $x^{*}:=x\left(\frac{1}{p}\right)$ and define integral degree bounds $d(v):=\left\lceil x^{*}(\delta(v))\right\rceil$.

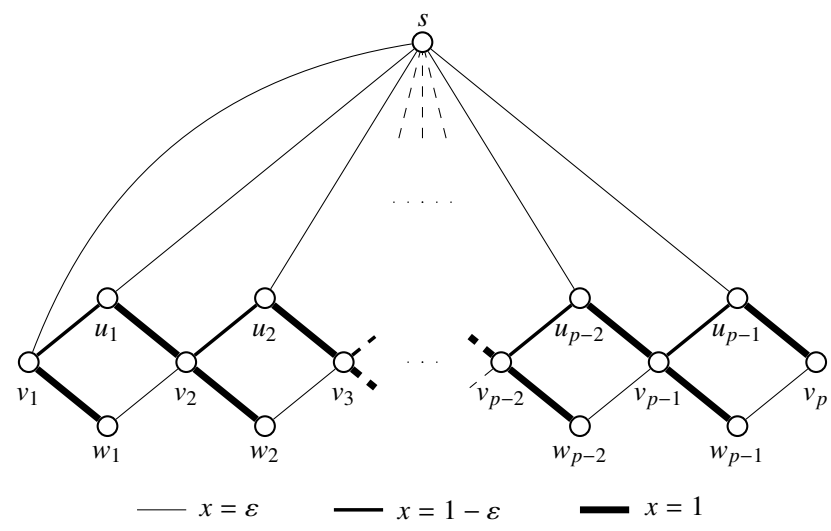

Figure 6: The graph $G=(V, E)$ and the points $x(\varepsilon) \in \mathbb{R}^{E}$, giving $x^{*}$ for $\varepsilon=\frac{1}{p}$.

We claim that $x^{*}$ is an extreme point of $P_{B D S T}(G, d)$. If so, the statement of Lemma 5 immediately follows because the degree bound slack at vertex $w_{1}$ is equal to

$$
d\left(w_{1}\right)-x^{*}\left(\delta\left(w_{1}\right)\right)=2-\left(1+\frac{1}{p}\right)=\frac{p-1}{p} .
$$


For proving that $x^{*}$ is an extreme point, we proceed as before. The point $x^{*}$ is feasible for $P_{S T}(G)$ because it can be written as a convex combination of spanning trees by averaging over the trees given in Figure 7. Feasibility for $P_{B D S T}(G, d)$ follows because the degree bounds are satisfied by definition.

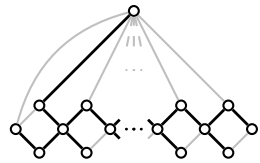

$T_{1}$

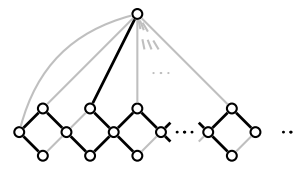

$T_{2}$

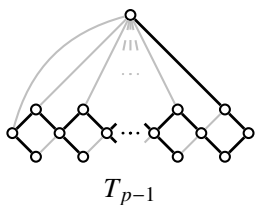

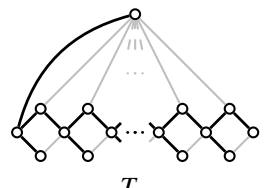

$T_{p}$

Figure 7: Trees $\left(T_{i}\right)_{i=1}^{p}$ for a convex combination giving $x^{*}$.

To see that $x^{*}$ is an extreme point of $P_{B D S T}(G, d)$, we show that $x^{*}$ is a unique solution of a system of tight constraints at $x^{*}$. Observe that for every vertex in the set $\left\{u_{1}, \ldots, u_{p-1}\right\} \cup$ $\left\{v_{1}, \ldots, v_{p-1}\right\}$, the corresponding degree constraint is tight at $x^{*}$. Moreover, it can be checked that the spanning tree constraints for all sets of the form $U_{i}:=\left\{u_{i}, v_{i}, v_{i+1}, w_{i}\right\}$, and for the endpoints of all edges of the form $u_{i} v_{i}$ or $v_{i} w_{i}$, are tight. These tight constraints are shown in Figure 8.

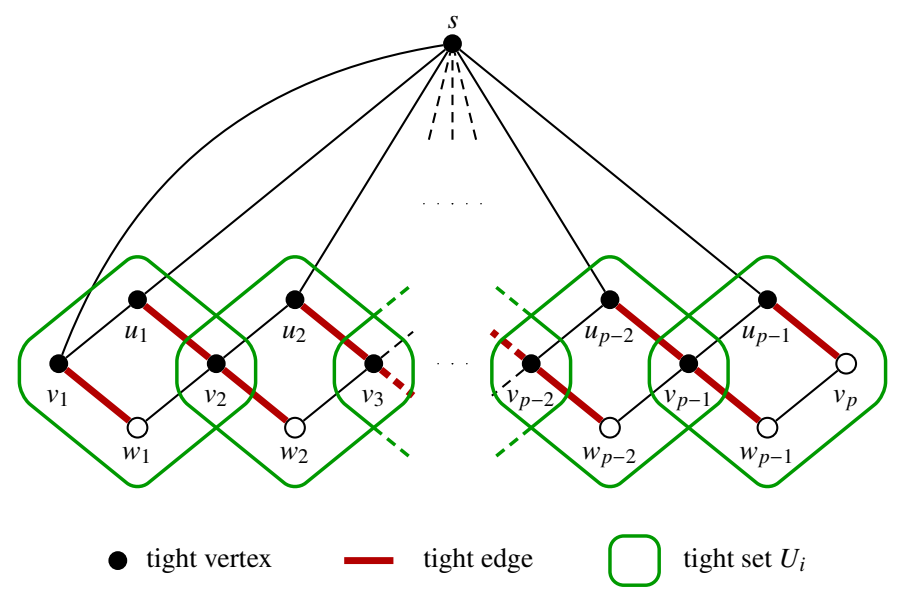

Figure 8: A selection of tight constraints for the point $x^{*} \in P_{B D S T}(G, d)$.

To show that $x^{*}$ is the unique solution of the system given by these tight constraints, we proceed similarly to the proof of Lemma 2. We show that under a certain ordering of the edges and the constraints, the matrix with indicator vectors of the constraints as rows has a special form. We use the order of the edges as indicated in Figure 9, and we number the constraints as follows, where $i \in[k-1]$, respectively:

Constraint 1: Degree constraint at $s$, containing the edges $e_{1}$, $e_{5}, e_{10}, \ldots, e_{5 p-5}$.

Constraint 5i-3: Tight edge $v_{i} u_{i}=e_{5 i-3}$.

Constraint $5 i-2$ : Tight edge $u_{i} v_{i+1}=e_{5 i-2}$.
Constraint $5 i-1$ : Degree constraint at $v_{i}$, containing the edges $e_{5 i-7}, e_{5 i-4}, e_{5 i-3}, e_{5 i-1}$ (and only the last three at $v_{1}$ ).

Constraint 5i: Degree constraint at $u_{i}$, containing the edges $e_{5 i-2}, e_{5 i-1}, e_{5 i}$.

Constraint $5 i+1$ : Tight set $U_{i}$, containing the edges $e_{5 i-3}$, $e_{5 i-2}, e_{5 i-1}, e_{5 i+1}$.

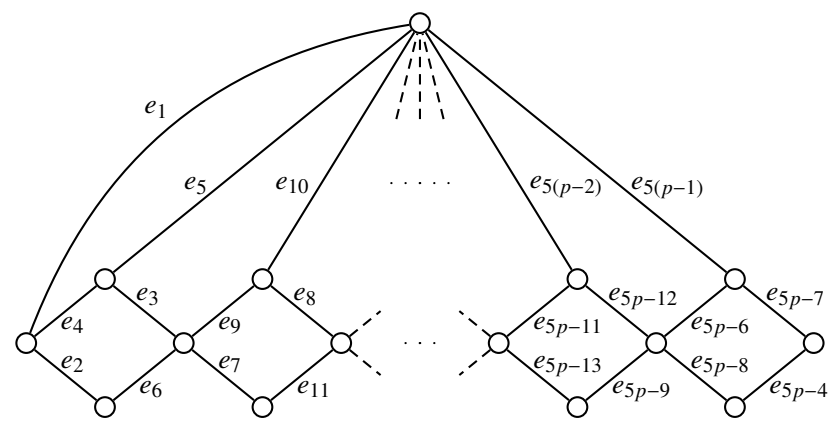

Figure 9: A numbering of the edges of $G$.

The different types of constraints (except for the tight edges) and the edges they contain are shown in Figure 10.<smiles>[Ge]C([As])([As])[As]</smiles>

$\delta\left(v_{i}\right)$

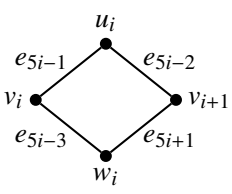

$E\left[U_{i}\right]$

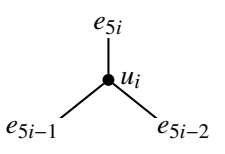

$\delta\left(u_{i}\right)$
Figure 10: Constraints and the underlying sets of edges.

Now consider the constraint matrix having the incidence vectors of the tight constraints as rows, where both the edges and the constraints are ordered as indicated. From the above list, it is easy to see that for $i \in\{2,3, \ldots, 5 k-4\}$, constraint $i$ contains edge $i$ and edges with lower index only. In other words, the square submatrix consisting of all but the first row and first column is lower triangular with ones on the diagonal, so this submatrix has full rank. Consequently, there is a onedimensional family of points $x \in \mathbb{R}^{E}$ satisfying all tight constraints but the first one, the degree constraint at $s$. It can be checked that this family is given by the points $x=x(\varepsilon) \in \mathbb{R}^{E}$ from Figure 6 , for $\varepsilon \in \mathbb{R}$. Imposing the degree constraint at $s$ on $x(\varepsilon)$, we get $1=x(\delta(s))=p \varepsilon$, hence the point $x\left(\frac{1}{p}\right)=x^{*}$ is indeed the unique solution of the system in question.

The next lemma shows that multiples of extremal degree slacks are extremal degree slacks as well.

Lemma 6. If $r \in \mathcal{D}$, then $k r \in \mathcal{D}$ for all $k \in \mathbb{Z}_{\geqslant 0}$.

Proof. We proceed by induction on $k$. Assume that $k r$ is an extremal degree slack and let $\left(G_{1}, d_{1}, x_{1}, v_{1}\right)$ and $\left(G_{2}, d_{2}, x_{2}, v_{2}\right)$ be certifying instances for the extremal degree slacks $r$ and $k r$. We show that $(k+1) r$ is an extremal degree slack.

The idea of the proof is similar to the proof of Lemma 3. We define the graph $G:=G_{1} \dot{\cup} G_{2} / v_{1} \sim v_{2}$, let $v=v_{1}=v_{2}$, and 
define a point $x \in \mathbb{R}^{E[G]}$ as well as degree bounds $d: V[G] \rightarrow \mathbb{Z}$ by

$$
\begin{aligned}
& \left.x\right|_{E\left[G_{1}\right]}:=x_{1}, \\
& \left.x\right|_{E\left[G_{2}\right]}:=x_{2} \text {, } \\
& \begin{aligned}
\left.d\right|_{V\left[G_{1}\right] \backslash\left\{v_{1}\right\}} & :=d_{1}, \\
\left.d\right|_{V\left[G_{2}\right] \backslash\left\{v_{2}\right\}} & :=d_{2}, \\
d(v) & :=d_{1}\left(v_{1}\right)+d_{2}\left(v_{2}\right) .
\end{aligned}
\end{aligned}
$$

Note that by assumption, for both $i \in\{1,2\}$, the degree constraint at $v_{i}$ in $P_{B D S T}\left(G_{i}, d_{i}\right)$ is not tight at $x_{i}$, so for each of the extreme points $x_{i}$, there exists a system of tight constraints with unique solution $x_{i}$ that does not contain the degree constraint at $v_{i}$. Copies of these constraints also appear in $P_{B D S T}(G, d)$, and the system of equalities that we obtain from these constraints has the unique solution $x$. Hence $x$ is an extreme point of $P_{B D S T}(G, d)$. Moreover, the slack of the degree bound at $v$ is

$$
\begin{aligned}
d(v)-x(\delta(v)) & =d_{1}\left(v_{1}\right)-x_{1}\left(\delta\left(v_{1}\right)\right)+d_{2}\left(v_{2}\right)-x_{2}\left(\delta\left(v_{2}\right)\right) \\
& =r+k r=(k+1) r,
\end{aligned}
$$

hence $(G, d, x, v)$ is a certifying instance for the extremal degree slack $(k+1) r$.

Combining the previous two lemmas is sufficient for proving Theorem 4, namely that every non-negative rational number is an extremal degree slack.

Proof of Theorem 4. By an earlier remark, we already know that $\mathbb{Z}_{\geqslant 0} \subseteq \mathcal{D}$. Let $\frac{p}{q}$, with coprime integers $p \geqslant 1$ and $q \geqslant 2$, be a positive rational number. We know that $r \in \mathcal{D}$ if and only if $\operatorname{frac}(r) \in \mathcal{D}$, so we can assume without loss of generality that $p<q$. By Lemma 5, $\frac{q-1}{q} \in \mathcal{D}$. Applying Lemma 6, we get that for any positive integer $k, \frac{k(q-1)}{q} \in \mathcal{D}$ and hence $\operatorname{frac}\left(\frac{k(q-1)}{q}\right) \in \mathcal{D}$ as well. Choosing $k$ such that $k(q-1) \equiv p(\bmod q)$, the latter fractional part equals $\frac{p}{q}$, proving $\frac{p}{q} \in \mathcal{D}$.

This concludes the construction of a family of counterexamples to Conjecture 1. By Theorem 4, every non-negative rational number is an extremal degree slack, so Lemma 3 allows modelling any rational degree bounds, in particular those required to model the instance from Lemma 2 containing the desired obstruction.

\section{Final Remarks}

The counterexample for Conjecture 1 is best possible in the following way. By a sparsity result of Goemans [8], any extreme point of ( $\left.\mathrm{LP}_{\mathrm{MBDST}}\right)$ is sparse in the sense that $|E[U]| \leqslant$ $2|U|-3$ for all $U \subset V$. Now recall that the obstruction constructed in Section 2 is a subgraph on $k$ vertices with $2 k-3$ edges on each of which the extreme point $x^{*}$ has a weight of $\varepsilon$. Consequently, the obstruction is densest possible while having arbitrarily little weight on it, as we can take $\varepsilon$ to be arbitrarily small by Theorem 4. Hence, apart from disproving Goemans' conjecture, the obtained obstruction is also a tight example for Goemans' sparsity result and shows that densest possible subgraphs within the support of a solution to (LP $\mathrm{PBDST}_{\mathrm{MBDT}}$ ) can have arbitrarily little $x^{*}$-weight.
Even though our counterexample shows a significant obstacle to improve Goemans' approximation algorithm for MBDST through a stronger partition matroid $M_{2}$ based on edge orientations, it remains open whether there may be a different matroid $M_{2}$ that can be used in Goemans' approach to obtain an approximation algorithm with a degree violation of only +1 .

\section{Acknowledgement}

The third author gratefully acknowledges the support of the Swiss National Science Foundation grant 200021_165866.

\section{References}

[1] M. Fürer, B. Raghavachari, Approximating the minimum-degree steiner tree to within one of optimal, Journal of Algorithms 17 (3) (1994) 409423. doi : 10.1006/jagm.1994.1042.

[2] R. Ravi, M. V. Marathe, S. S. Ravi, D. J. Rosenkrantz, H. B. Hunt III, Many birds with one stone: Multi-objective approximation algorithms, in: 25th Annual ACM Symposium on Theory of Computing, ACM, 1993, pp. 438-447. doi : 10.1145/167088.167209.

[3] R. Ravi, M. V. Marathe, S. S. Ravi, D. J. Rosenkrantz, H. B. Hunt, Approximation algorithms for degree-constrained minimum-cost networkdesign problems, Algorithmica 31 (1) (2001) 58-78. doi:10.1007/ s00453-001-0038-2.

[4] J. Könemann, R. Ravi, A matter of degree: Improved approximation algorithms for degree-bounded minimum spanning trees, in: 32nd Annual ACM Symposium on Theory of Computing, ACM, 2000, pp. 537-546. doi: 10.1145/335305.335371.

[5] J. Könemann, R. Ravi, Primal-dual meets local search: Approximating mst's with nonuniform degree bounds., in: 35th Annual ACM Symposium on Theory of Computing, ACM, 2003, pp. 389-395. doi: 10.1145/780542.780600.

[6] K. Chaudhuri, S. Rao, S. Riesenfeld, K. Talwar, A push-relabel approximation algorithm for approximating the minimum-degree MST problem and its generalization to matroids, Theoretical Computer Science 410 (2009) 4489-4503. doi : 10.1016/j.tcs . 2009.07.029.

[7] K. Chaudhuri, S. Rao, S. Riesenfeld, K. Talwar, What would Edmonds do? Augmenting paths and witnesses for degree-bounded MSTs, Algorithmica 55 (2009) 157-189. doi : 10.1007/s00453-007-9115-5.

[8] M. X. Goemans, Minimum bounded degree spanning trees, in: 47th Annual IEEE Symposium on Foundations of Computer Science, IEEE, 2006, pp. 273-282. doi : 10.1109/FOCS. 2006. 48.

[9] M. Singh, L. C. Lau, Approximating minimum bounded degree spanning trees to within one of optimal, in: 39th Annual ACM Symposium on Theory of Computing, ACM, 2007, pp. 661-670. doi : 10.1145/1250790. 1250887.

[10] N. Bansal, R. Khandekar, V. Nagarajan, Additive guarantees for degreebounded directed network design, SIAM Journal on Computing 39 (4) (2009) 1413-1431. doi:10.1137/080734340.

[11] C. Chekuri, J. Vondrák, R. Zenklusen, Dependent randomized rounding via exchange properties of combinatorial structures, in: Proceedings of the 51st IEEE Symposium on Foundations of Computer Science (FOCS), 2010, pp. 575-584. doi:10.1109/FOCS. 2010.60.

[12] N. Bansal, R. Khandekar, J. Könemann, V. Nagarajan, B. Peis, On generalizations of network design problems with fegree bounds, Mathematical Programming, Series A 141 (2013) 479-506. doi:10.1007/ s10107-012-0537-8.

[13] R. Zenklusen, Matroidal degree-bounded minimum spanning trees, in: Proceedings of the 23rd Annual ACM-SIAM Symposium on Discrete Algorithms (SODA), 2012, pp. 1512-1521.

[14] N. Olver, R. Zenklusen, Chain-constrained spanning trees, in: Proceedings of the 16th International Conference on Integer Programming and Combinatorial Optimization (IPCO), 2013, pp. 324-335. doi:10.1007/ 978-3-642-36694-9_28.

[15] A. Linhares, C. Swamy, Approximating min-cost chain-constrained spanning trees: A reduction from weighted to unweighted problems, in: Proceedings of the 18th Conference on Integer Programming and Combinatorial Optimization (IPCO), 2016.

[16] A. Schrijver, Combinatorial optimization: Polyhedra and efficiency, Vol. 24, Springer, 2003. 